\title{
Acantholytic Anaplastic Extramammary Paget's Disease: A Case Report and Review of the Literature
}

\author{
Yu-Jin Oh, M.D., Bark-Lynn Lew, M.D., Ph.D., Woo-Young Sim, M.D., Ph.D. \\ Department of Dermatology, College of Medicine, Kyung Hee University, Seoul, Korea
}

Extramammary Paget's disease (EMPD) is an uncommon intraepithelial neoplasm that most commonly arises on the vulva and perianal region. Very few cases of EMPD revealing a histological Bowenoid appearance have been reported. This study describes scrotal EMPD presenting with histological features of Bowen's disease in a 79-year-old man. He presented with a 5-year history of a pruritic erythematous plaque and a verrucous papule on the scrotum. The verrucous papule histopathologically showed Bowenoid features, and the erythematous plaque demonstrated acantholytic EMPD. Immunohistochemical findings revealed strong expression for carcinoembryonic antigen, Cam 5.2, epithelial membrane antigen, cytokeratin (CK) 7, and pancytokeratin (AE1/AE3) in both areas, but negative CK20 staining, supporting the overall diagnosis of primary acantholytic anaplastic EMPD. This is the first reported case of acantholytic anaplastic EMPD in the Korean literature. (Ann Dermatol 23(S2) S226 S230, 2011)

\section{-Keywords-}

Acantholytic, Anaplastic, Bowenoid, Extramammary Paget disease, Immunohistochemistry

Received April 5, 2011, Revised June 7, 2011, Accepted for publication June 14, 2011

Corresponding author: Bark-Lynn Lew, M.D., Ph.D., Department of Dermatology, Kyung Hee University Hospital at Gang-dong, 149 Sangil-dong, Gangdong-gu, Seoul 134-727, Korea. Tel: 82-2-440-7329, Fax: 82-2-440-7336, E-mail: bellotte@hanmail.net

This is an Open Access article distributed under the terms of the Creative Commons Attribution Non-Commercial License (http:// creativecommons.org/licenses/by-nc/3.0) which permits unrestricted non-commercial use, distribution, and reproduction in any medium, provided the original work is properly cited.

\section{INTRODUCTION}

The histological resemblance between extra-mammary Paget's disease (EMPD) and Bowen's disease has been described since Bowen's original article was published in 1912. Very few cases of EMPD revealing a histological Bowenoid appearance have been reported. We describe a case of scrotal EMPD presenting with histological features of Bowen's disease and review the related literature.

\section{CASE REPORT}

A 79-year-old man visited our hospital for scrotal skin lesions with itching for 5 years. Two well-circumscribed lesions were evident on the scrotum. One was a solitary verrucous surfaced papule, and the other was a localized, well-defined erythematous plaque with yellowish scales (Fig. 1). Nothing was remarkable in his medical history or laboratory findings.

A skin biopsy from the solitary papule showed full- thick-

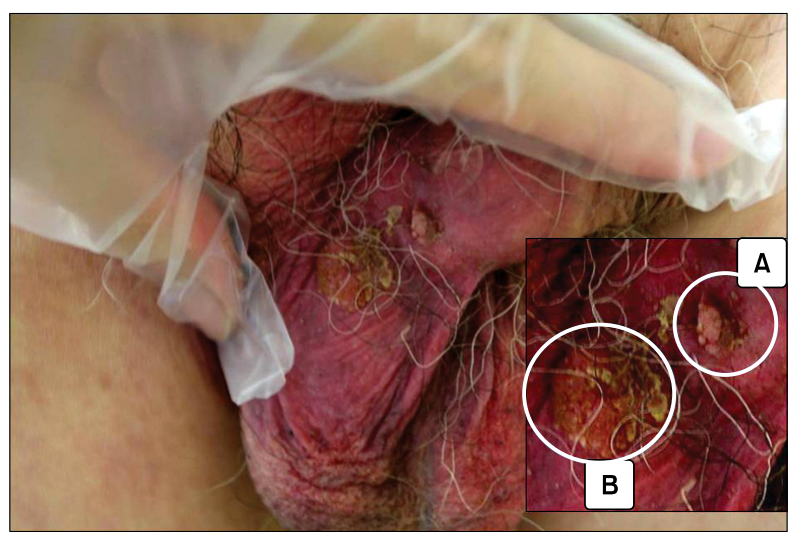

Fig. 1. (A) A solitary verrucous surfaced, sessile papule was observed on the scrotum. (B) A localized, well defined, erythematous plaque with yellow scales was observed on the scrotum. 

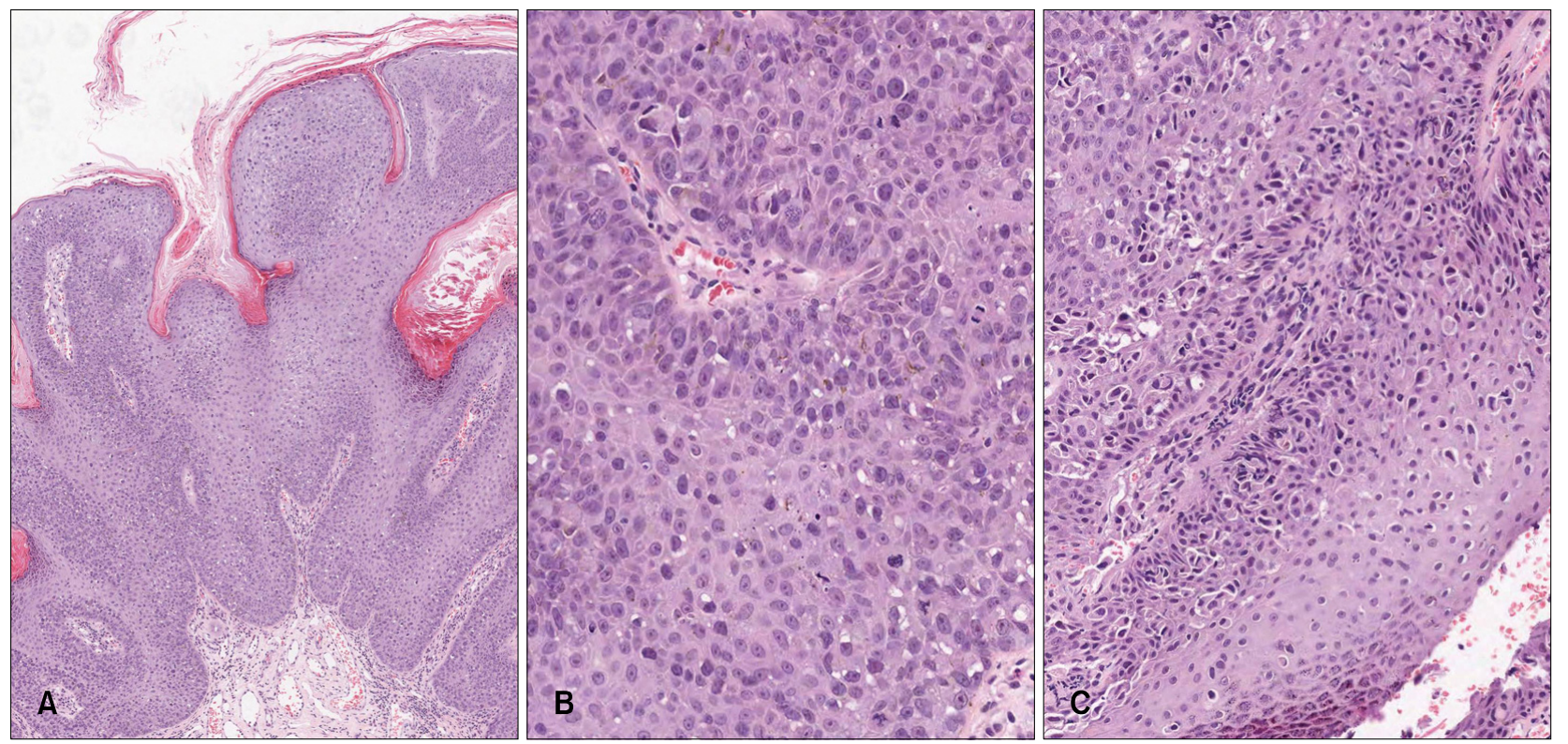

Fig. 2. Specimen obtained from the papular lesion. (A) The epidermis revealed marked acanthosis with papillomatosis $(\mathrm{H} \& \mathrm{E}, \times 100)$. (B) Full thickness cellular atypia with mitotic figures was observed $(\mathrm{H} \& \mathrm{E}, \times 200)$. (C) Atypical pleomorphic cells found in the spinous zone, a few of which had abundant, pale cytoplasm, were separated by clefts from the surrounding cells $(H \& E, \times 200)$.
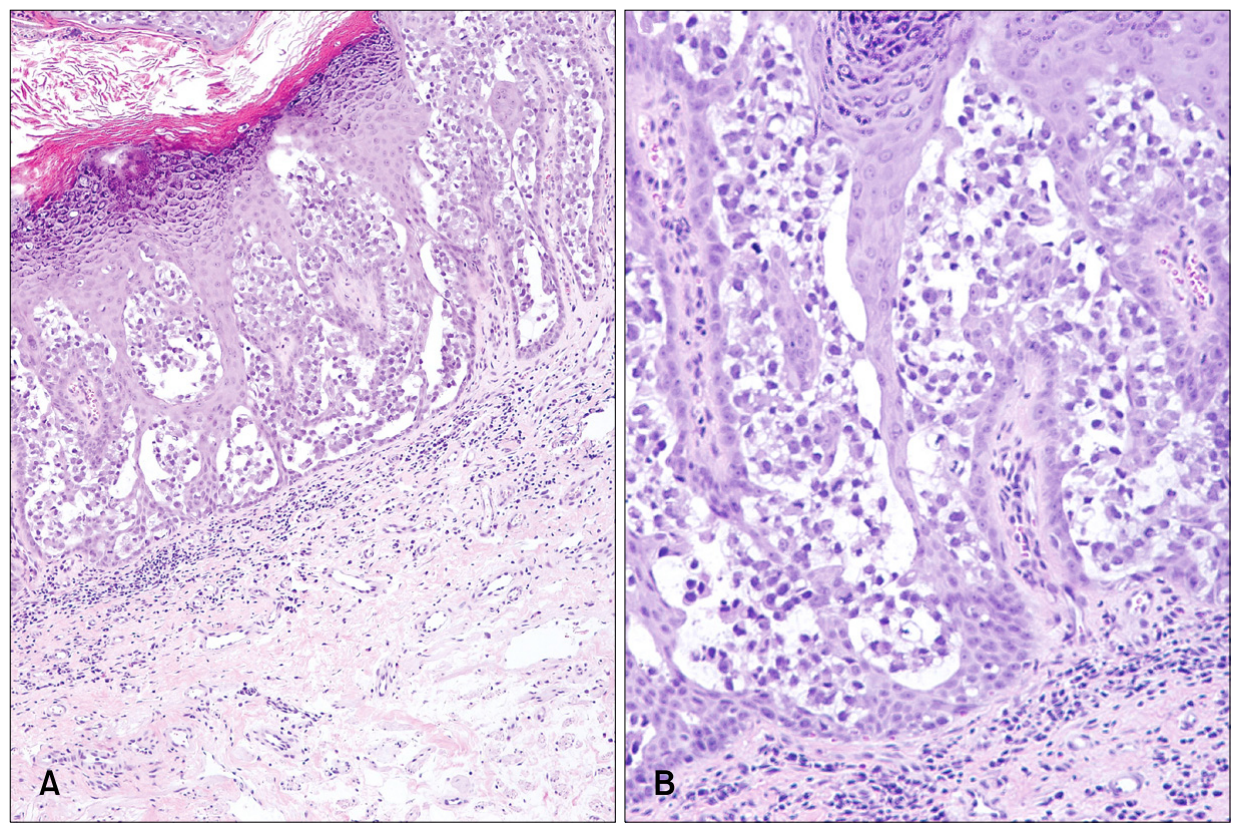

Fig. 3. Specimen obtained from the plaque lesion. (A) The epidermis showed prominent acantholysis with nests of Paget cells in the cavity $(\mathrm{H} \& \mathrm{E}, \times 100)$. (B) The Paget cells had pale-staining cytoplasm with vesicular nuclei containing occasional prominent nucleoli and were separated from the underlying dermis by flattened basal cells $(H \& E, \times 200)$. ness cellular atypia with mitotic figures, similar to Bowen's disease. The atypical pleomorphic cells found in the spinous zone of different foci, in which a few cells had abundant, pale cytoplasm, were separated by clefts from the surrounding cells (Fig. 2). The other specimen was obtained from the yellowish erythematous plaque. The epidermis showed prominent acantholysis with nests of Paget cells in the cavity. The Paget cells had pale-staining cyto- plasm with vesicular nuclei containing occasional prominent nucleoli and were separated from the underlying dermis by flattened basal cells (Fig. 3).

Carcinoembryonic antigen (CEA), Cam 5.2, epithelial membrane antigen, cytokeratin (CK) 7, and pancytokeratin (AE1/AE3) were strongly expressed in both areas based on immunohistochemical stains. Gross cystic disease fluid protein (GCDFP-15) was also strongly expressed 

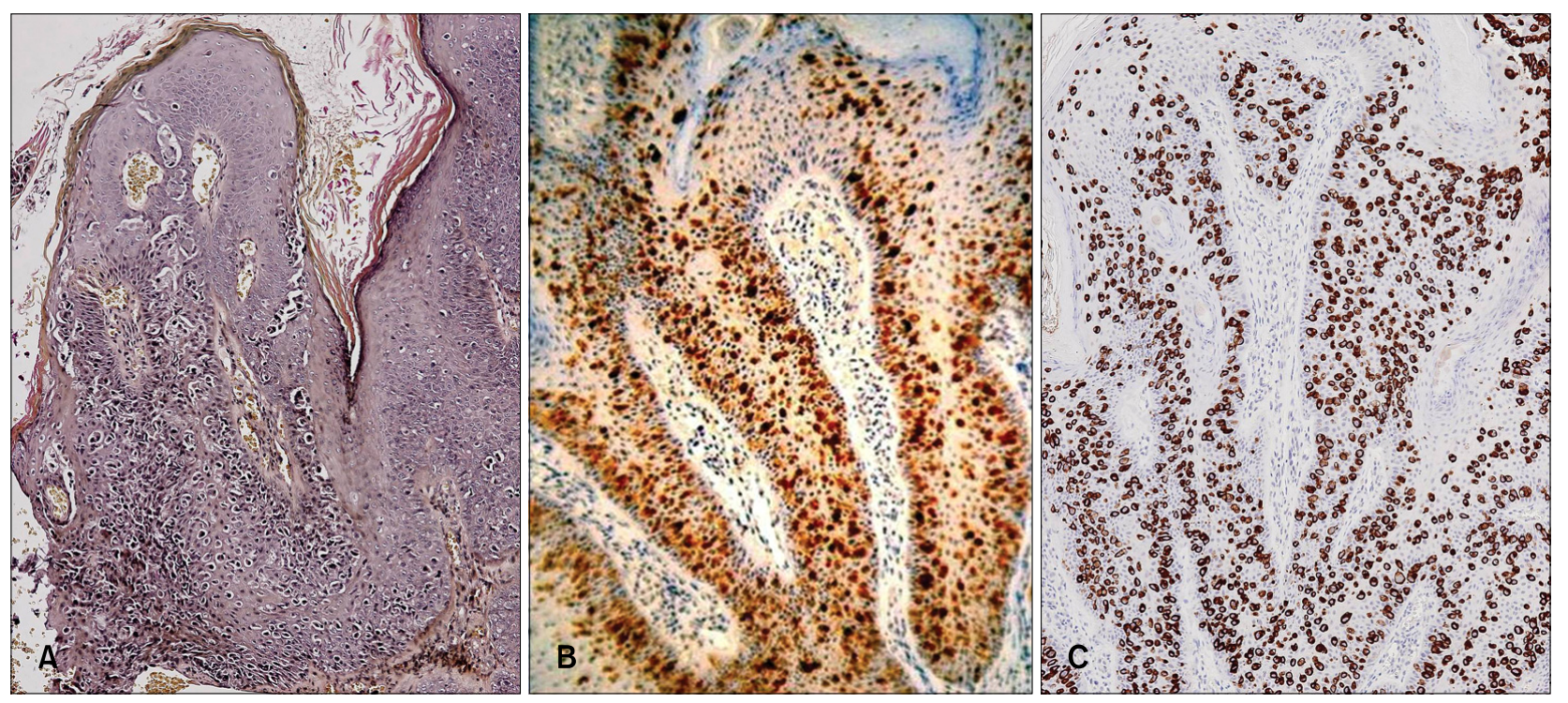

Fig. 4. Immunohistochemical stains of the papular lesion. (A) Mucicarmine, (B) Carcinoembryonic antigen, (C) Cytokeratin 7 (magnification, $\times 100$ ).
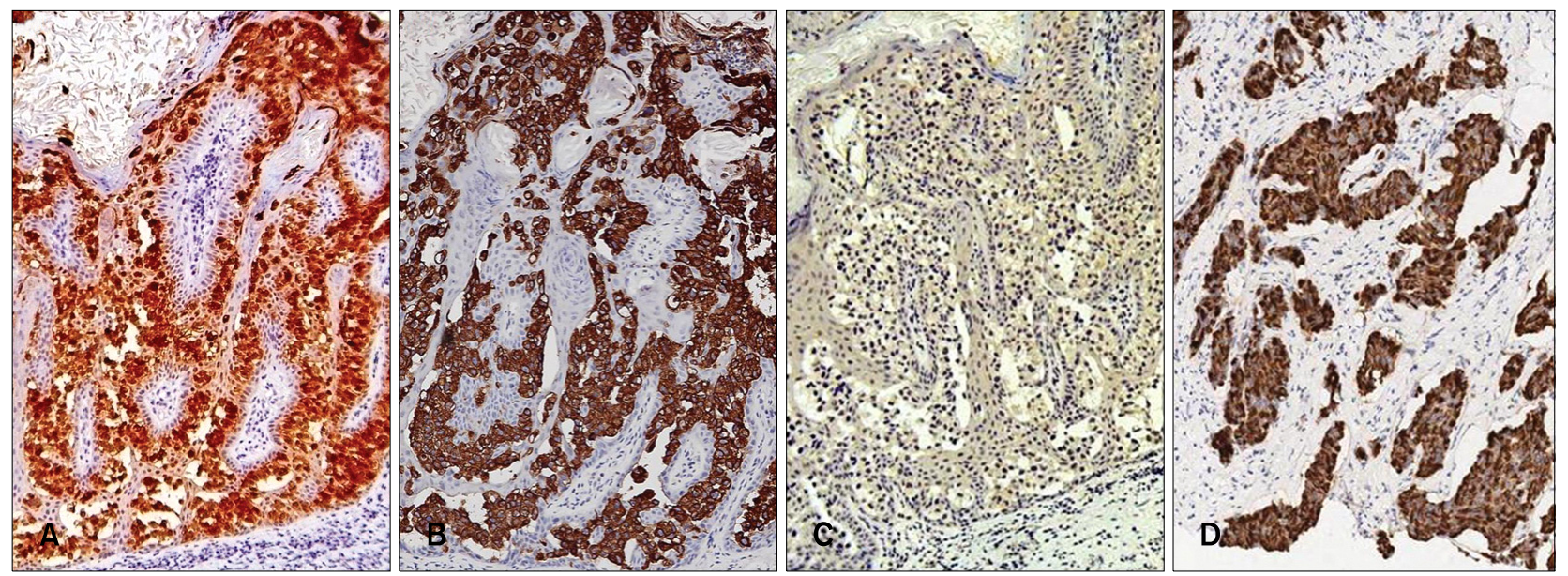

Fig. 5. Immunohistochemical stains of the plaque lesion. (A) Carcinoembryonic antigen, (B) Cytokeratin 7, (C) CK20, (D) Gross cystic disease fluid protein (magnification, $\times 100$ ).

in the Paget cells. However, staining for mucicarmine, diastase-resistant periodic acid-Schiff (d- PAS), and alcian blue were not positive in the area with Bowenoid appearance but were observed in the area with Paget cells. Staining for CK20 was completely negative in both areas (Fig. 4, 5).

Additionally, we measured serum tumor markers such as CEA, carbohydrate antigen, $\alpha$-fetoprotein, and prostatespecific antigen and found nothing remarkable except that CEA had increased by $5.2 \mathrm{ng} / \mathrm{ml}$. Abdominal ultrasonography and positron emission tomography-computed tomography were performed to rule out EMPD associated with an internal malignancy, but no evidence of internal malignancy or metastasis was found. Thus, we diagnosed primary acantholytic anaplastic EMPD. Because the patient's age and general condition were considered, and he did not have any internal malignancy, no further treatment was needed after a wide local excision $(0.5 \mathrm{~cm}$ margin). He had regular follow-up evaluations to detect recurrence.

\section{DISCUSSION}

Classic EMPD can be diagnosed relatively easily by histopathological examination. However, it is impossible to distinguish EMPD and Bowen's disease solely through a routine histological examination, because clear cells can be observed in both diseases, and anaplastic EMPD may 
be a diagnostic pitfall.

The concept of Pagetoid Bowen disease was first described by Williamson et al. ${ }^{1}$ as a histological variant of squamous cell carcinoma in situ that shows nests of cells with pale-staining cytoplasm within the epidermis. That is, this disease has clear cells but lacks the characteristic immunohistochemical features of EMPD. Additionally, EMPD also exhibits histological features of Bowen disease $^{2,3}$. A few cases have been reported, and most have involved the vulva.

A rare and less-known variant of Paget's disease (PD), which was originally named anaplastic PD by Rayne and Santa Cruz, has only been reported twice in the literature $^{4,5}$, but it has not been reported as anaplastic EMPD. This less-known variant is similar to Bowen's disease in full-thickness cellular atypia without conventional Paget's cells. However, its distinguishing features are acantholysis and the absence of dyskeratotic cells. Immunohistochemically, neoplastic cells are negative for mucin stains such as d-PAS, mucicarmine, and alcian blue; however, they stain positively for CK7 and CAM 5.2, as expected in classic PD. There are no previous reports of anaplastic EMPD.

The hypothesis that Paget cells are derived from apocrine glands in many cases of EMPD is most widely accepted and is supported by immunohistochemical studies that show strong CEA expression in Paget cells ${ }^{3}$. Mazoujian et al. ${ }^{6}$ suggested that GCDFP-15 was more specific evidence for an apocrine gland origin. Therefore, our findings support the hypothesis that EMPD arises from the malignant transformation of basal stem cells that undergo apocrine gland differentiation ${ }^{2}$. Staining for CK20 is positive in secondary EMPD and negative in primary EMPD. Both lesions in the current case were negative to CK20, so we finally diagnosed primary EMPD.

Four cases of combined EMPD with Bowen's disease have been reported ${ }^{2,7-9}$. Hawley et al. ${ }^{7}$ reported a case of a 66-year-old female who had clinical features of EMPD on her vulva. Histopathologically, Paget cells and intraepithelial neoplasia were mixed: Paget cells were CEA positive, but atypical keratinocytes were negative.

Orlandi et al. $^{8}$ reported a case of a 66 -year-old female who had a clinical Bowen-like lesion on her vulva. A biopsy was performed, and the result revealed keratinizing vulval intraepithelial neoplasia admixed with EMPD. Paget cells were positive for CEA and CK8, whereas the atypical keratinocytes were negative for CEA. Quinn et al. ${ }^{2}$ presented the case of a 60-year-old man who was previously diagnosed with squamous cell carcinoma in situ of the scrotum. Specimens obtained from the lesion revealed EMPD adjacent to areas characteristic of Bowen's disease. This was the first case reported as scrotal EMPD with features of Bowen's disease. The case of a 61-yearold female with pruritic erythematous lesion on the vulva was reported by Matsumoto et al. ${ }^{9}$ A histopathological examination revealed that Bowen's disease and EMPD were mixed but sharply separated. Paget cells were positive for CEA, CK7 and 8, whereas atypical keratinocytes were negative for CEA but positive for CK7 and $8^{9}$. They assumed that CEA was the most reliable marker to identify EMPD or Bowen's disease. Furthermore, strong CK7 and 8 expression in both Paget cells and keratinocytes suggested that these lesions were derived from a common cellular origin.

In the present case, the papular lesion exhibited a Bowenoid appearance; however, based on cytological atypia and immunohistochemical findings in different foci, we finally diagnosed acantholytic anaplastic EMPD. The erythematous plaque lesion was diagnosed as acantholytic EMPD by both histopathological and immunohistochemical evidence.

Anaplastic PD is a rare subtype of PD, as there are no previous reports of anaplastic EMPD. It may be mistaken for Bowen's disease based on the unusual histopathological features not seen in cases of conventional EMPD. Therefore, in cases of suspicious EMPD, an appropriate immunohistochemical study can lead to an accurate diagnosis and treatment. Furthermore, a solitary verrucous papule, as seen in our case, is a rare and interesting clinical finding of EMPD. Our findings support the hypothesis that primary EMPD arises multifocally from multipotent epidermal cells.

\section{REFERENCES}

1. Williamson JD, Colome MI, Sahin A, Ayala AG, Medeiros LJ. Pagetoid bowen disease: a report of 2 cases that express cytokeratin 7. Arch Pathol Lab Med 2000;124:427-430.

2. Quinn AM, Sienko A, Basrawala Z, Campbell SC. Extramammary Paget disease of the scrotum with features of Bowen disease. Arch Pathol Lab Med 2004;128:84-86.

3. Cannavò SP, Guarneri F, Napoli P. Extramammary Paget's disease of the scrotum with Bowenoid features. Eur J Dermatol 2006;16:203-204.

4. Rayne SC, Santa Cruz DJ. Anaplastic Paget's disease. Am J Surg Pathol 1992;16:1085-1091.

5. Mobini N. Acantholytic anaplastic Paget's disease. J Cutan Pathol 2009;36:374-380.

6. Mazoujian G, Pinkus GS, Haagensen DE Jr. Extramammary Paget's disease--evidence for an apocrine origin. An immunoperoxidase study of gross cystic disease fluid protein-15, carcinoembryonic antigen, and keratin proteins. Am J Surg Pathol 1984;8:43-50.

7. Hawley IC, Husain F, Pryse-Davies J. Extramammary Paget's 
disease of the vulva with dermal invasion and vulval intra-epithelial neoplasia. Histopathology 1991;18:374-376.

8. Orlandi A, Piccione E, Sesti F, Spagnoli LG. Extramammary Paget's disease associated with intraepithelial neoplasia of the vulva. J Eur Acad Dermatol Venereol 1999;12:183-185.
9. Matsumoto $M$, Ishiguro $M$, Ikeno $F$, Ikeda $M$, Kamijima $R$, Hirata $Y$, et al. Combined Bowen disease and extramammary Paget disease. J Cutan Pathol 2007;34 Suppl 1:47-51. 